\title{
Husain-Kuchař model: Time variables and nondegenerate metrics
}

\author{
J. Fernando Barbero G., Alfredo Tiemblo, and Romualdo Tresguerres \\ Centro de Física “Miguel Catalán,', Instituto de Matemáticas y Física Fundamental, CSIC, Serrano 113 bis, 28006 Madrid, Spain
}

(Received 26 November 1997; published 24 April 1998)

\begin{abstract}
We study the Husain-Kuchař model by introducing a new action principle similar to the self-dual action used in the Ashtekar variables approach to quantum gravity. This new action has several interesting features, among them the presence of a scalar time variable that allows the definition of geometric observables without adding new degrees of freedom, the appearance of a natural nondegenerate four-metric, and the possibility of coupling ordinary matter. [S0556-2821(98)06310-3]
\end{abstract}

PACS number(s): 04.20.Cv, 04.20.Fy

\section{INTRODUCTION}

In the long quest to understand general relativity (GR) the use of toy models has a long tradition. This is especially true in quantum gravity and quantum cosmology where they have allowed us to obtain some, otherwise very difficult to get, information. However, this does not come without a price because one is usually forced to introduce very strong simplifying assumptions and, quite often, some of the key features of the theory are lost. Though a final judgement on the success of this approach can only be made once a consistent quantum gravity theory is found, it is possible, in principle, to get some clues on how well one is doing by considering widely different toy models.

Bianchi models (see, for example, Ref. [1]) are obtained by imposing homogeneity conditions on the gravitational variables. Their high symmetry has the consequence of killing most of the degrees of freedom of the full theory leaving only a finite number of them. They have been widely used in quantum cosmology mainly because the equations obtained upon quantization are more or less tractable.

There are other (less known) toy models that achieve the goal of simplifying the theory by going in the opposite direction: adding degrees of freedom. Chief among them is the Husain-Kuchăr (HK) model [2]. This model is quite interesting because it has some of the features that make GR so difficult to deal with in the quantum regime, in particular diffeomorphism invariance, but is significantly simpler because it lacks the Hamiltonian constraint (another important source of difficulties in full GR). This has the effect of increasing the number of degrees of freedom per space point from 2 to 3 .

To illustrate with a picture the different and complementary roles played by these two approaches one can make the following analogy. Portray GR as a complicated, knotted, two-dimensional surface $\Sigma$ embedded in $\mathbb{R}^{3}$. Working with Bianchi models is something akin to trying to get information about $\Sigma$ by looking at a finite number of points on it. The HK model, on the other hand, is like trying to gather information by studying the whole $\mathbb{R}^{3}$. Clearly some crucial features are lost in both approaches but, still, they provide useful and complementary views about $\Sigma$. ${ }^{1}$

The HK model, in its usual formulation (see Ref. [3] for some alternative descriptions), can be conveniently derived from an action principle very close to the self-dual action [4] from which the Ashtekar approach to classical and quantum GR [5] can be found. The phase space of the Hamiltonian description of both theories is the same: it is coordinatized by a $\mathrm{SO}(3)$ connection and a densitized (inverse) triad canonically conjugate to it. Their crucial difference is the absence of a Hamiltonian constraint in the HK model. The usual interpretation of this lack of "dynamics" is the following. By using the frame field in terms of which the HK action is written ${ }^{2}$ one can build a degenerate four-metric $g_{a b}$ and a densitized vector field $\widetilde{n}^{a}$ (that can be dedensitized by means of an auxiliary space-time foliation). The lack of dynamics can be seen as the fact that the Lie derivative of $g_{a b}$ in the direction of $n^{a}$ is zero.

The four-dimensional metric that we can build from the frame field in the HK action is degenerate. This can lead to the erroneous conclusion that the model describes only degenerate four-metrics; a fact that has induced some authors to claim, for example, that ordinary matter cannot be coupled to the model. We will show that this is not the case in due time but at this point we urge the reader to think about the following paradoxical situation. The fact that the Hamiltonian constraint is missing from the $\mathrm{HK}$ model means that the constraint hypersurface of GR in the Ashtekar formulation is contained in the HK one, hence, every solution to GR (for example, Minkowski space-time) is a solution to the HK model. How can we then describe these GR solutions in terms of the fields present in the HK action if we only have a $4 \times 3$ frame field available?

The solution to this problem that we give in the paper has some unexpected implications that make it quite attractive.

\footnotetext{
${ }^{1}$ This analogy is, actually, a little bit more than that because the Hamiltonian formulation of GR can be understood as the study, in phase space, of the hypersurface defined by the constraints.

${ }^{2}$ Being a $4 \times 3$ matrix it is neither a tetrad nor a triad.
} 
On one hand it provides an elegant way to define quantum geometric observables (such as areas and volumes) without having to resort to increasing the number of physical degrees of freedom as in previous approaches [6,7]. On the other, it allows the introduction of a kind of time variable in the double sense that dynamics can be referred to it and also that the scalar constraint (that we need now in order to get the correct counting of degrees of freedom) is linear in its canonically conjugate momentum (so that, upon quantization it gives a Schrödinger-type of equation).

The main result of the paper is that it is possible to obtain the HK model from an action principle (also related to the self-dual action) that admits an interpretation in terms of nondegenerate four-dimensional metrics. This is achieved by introducing a scalar field that can be interpreted, in a sense that will be made more precise later, as the time variable mentioned before. This will not only solve the paradox presented above but also will provide a means to couple ordinary matter thus enhancing the usefulness of $\mathrm{HK}$ as a toy model. We hope that the possible interpretation of this scalar field as time will help to shed some light on the problem of time in full GR.

The paper is organized as follows. This introduction is followed by Sec. II where the usual formulation of the Husain-Kuchar model is briefly reviewed. The new action principle, which is the object of this paper, is introduced in Sec. III where we derive it from the well known self-dual action for GR. The details of the Hamiltonian formulation of our model are spelled out in Sec. IV. There we thoroughly study the derivation of the constraints of the theory and discuss their interpretation. In Sec. V we compare the field equations in both the usual and the new formulation for the HK model in order to show that they are not in contradiction (a nontrivial fact as the number of equations is different in both cases). Section VI gives a different proof of the equivalence of our "nondegenerate" formulation and the usual one at the Lagrangian level. We also show that the addition of a cosmological constant (made possible in our scheme by the availability of a nondegenerate four-metric) does not lead us beyond the HK model. We end the paper with Sec. VII, where we give our conclusions and general comments, and an appendix that contains some details of the computations needed to disentangle the constraints in our formulation.

\section{THE HUSAIN-KUCHAŘ MODEL: A BRIEF REVIEW}

We review in this section the HK model in its usual formulation in order to describe its main features and collect the most important formulas for future reference. We start from the action [2]

$$
S=\frac{1}{2} \int_{\mathcal{M}} d^{4} x \widetilde{\eta}^{a b c d} \epsilon_{i j k} e_{a}^{i} e_{b}^{j} F_{c d}^{k},
$$

where our notation is the following: $\mathcal{M}$ is a four-dimensional manifold $\mathcal{M}=\mathbb{R} \times \Sigma$ with $\Sigma$ a three-dimensional manifold (that we take compact and without boundary so that we can freely integrate by parts). Curved space-time indices are represented by lower case Latin letters from the beginning of the alphabet. We will make no distinction between fourdimensional and three-dimensional indices. The dimension- ality of a certain field will be clear from the context. The three- and four-dimensional Levi-Civita tensor densities will be denoted as $\widetilde{\eta}^{a b c}$ and $\widetilde{\eta}^{a b c d}$, respectively $\left(\eta_{a b c}\right.$ and $\eta_{a b c d}$ are their inverses). We use the convention of representing the density weights of geometrical objects by using tildes above (positive) and below (negative) the stem letter representing them. Internal $\mathrm{SO}(3)$ indices, running from 1 to 3 will be denoted by Latin letters from the middle of the alphabet and the internal Levi-Civita tensor as $\epsilon^{i j k}$. We will also use a $\mathrm{SO}(3)$ connection $A_{a}^{i}(x)$ that defines a covariant derivative acting on internal indices as $\nabla_{a} \lambda_{i}=\partial_{a} \lambda_{i}+\epsilon_{i j k} A_{a}^{j} \lambda^{k}$ and can be extended to space-time indices by using any torsion-free space-time connection; none of the results that we present in the paper will depend on the extension chosen. The curvature of $A_{a}^{i}(x)$ is defined as $F_{a b}^{i}=2 \partial_{[a} A_{b]}^{i}+\epsilon_{j k}^{i} A_{a}^{j} A_{b}^{k}$. The frame field $e_{a}^{i}$ in the previous action is a $4 \times 3$ matrix; we will reserve the name triad for its projection on the threedimensional slices used in the Hamiltonian formalism.

The field equations derived from Eq. (1) are

$$
\begin{gathered}
\epsilon_{i j k} e_{[b}^{j} F_{c d]}^{k}=0, \\
\epsilon_{i j k} e_{[b}^{j} \nabla_{c} c_{d]}^{k}=0 .
\end{gathered}
$$

Some interesting features of Eq. (2) are summarized in the following formulas:

$$
\begin{gathered}
\tilde{n}^{a} F_{a b}^{i}=0, \\
\tilde{n}^{a} \nabla_{[a} e_{b]}^{i}=0, \\
\mathcal{L}_{n^{a}}\left(e_{a}^{i} e_{b i}\right)=0,
\end{gathered}
$$

where $\widetilde{n}^{a}=(1 / 3 !) \widetilde{\eta}^{a b c d} \epsilon_{i j k} e_{b}^{i} e_{c}^{j} e_{d}^{k}, n^{a}=\widetilde{n}^{a} / \widetilde{e}$, and $\widetilde{e}$ is defined by means of an auxiliary foliation defined by a scalar function $t$ as $\widetilde{e} \equiv \widetilde{n}^{a} \partial_{a} t$. $\mathcal{L}_{n^{a}}$ denotes the Lie derivative along the direction defined by $n^{a}$. The first two equations in Eq. (3) explain why we do not have a dynamics in the model [2] (the projections of the field equations on to the direction normal to the spatial slices are zero) while the last one, which is a consequence of the others, displays this lack of evolution as the fact that the Lie derivative of the degenerate four-metric $e_{a}^{i} e_{b i}$ along $n^{a}$ is zero.

The meaning of this model is best understood in the Hamiltonian framework. In order to define it we introduce a foliation by means of a scalar function $t$ and a congruence of curves (nowhere tangent to the surfaces of the foliation) parametrized by $t$ whose tangent vectors we denote $t^{a}$. By doing this we have that $t^{a} \partial_{a} t=1$ and, hence, the time derivatives can be interpreted as the Lie derivatives along the direction defined by $t^{a}$. We can write Eq. (1) as

$$
\begin{aligned}
S= & \int d t \int_{\Sigma} d^{3} x\left\{\dot{A}_{a}^{i}\left[\widetilde{\eta}^{a b c} \epsilon_{i j k} e_{b}^{j} e_{c}^{k}\right]+A_{0}^{i} \nabla_{a}\left[\widetilde{\eta}^{a b c} \epsilon_{i j k} e_{b}^{j} e_{c}^{k}\right]\right. \\
& \left.+e_{0}^{i}\left[\widetilde{\eta}^{a b c} \epsilon_{i j k} e_{a}^{j} F_{b c}^{k}\right]\right\}
\end{aligned}
$$

where the overdots denote time derivatives of the fields (Lie derivatives along the direction defined by $\left.t^{a}\right), A_{0}^{i} \equiv t^{a} A_{a}^{i}$, and $e_{0}^{i} \equiv t^{a} e_{a}^{i}$. After following the usual Dirac procedure [8] one finds out that the phase space of the model is coordinatized 
by a $\mathrm{SO}(3)$ connection $A_{a}^{i}$ and a canonically conjugate densitized triad $\widetilde{E_{i}^{a}}$. The first class constraints are

$$
\begin{aligned}
& \nabla_{a} \widetilde{E}_{i}^{a}=0, \\
& \widetilde{E}_{i}^{a} F_{a b}^{i}=0 .
\end{aligned}
$$

The first constraint (Gauss law) generates internal $\mathrm{SO}(3)$ rotations whereas the second (known as vector constraint) generates spatial diffeomorphisms. ${ }^{3}$ As we can see there is no scalar constraint so that we have three degrees of freedom per space point.

\section{FROM THE SELF-DUAL ACTION TO THE HUSAIN-KUCHA R MODEL}

In this section we introduce a modified action principle for the Husain-Kuchar model that allows us to use fourdimensional, nondegenerate metrics in order to describe it. We take as the starting point the self-dual action ${ }^{4}$ of Samuel, Jacobson, and Smolin [4]

$$
S=-\frac{1}{2} \int_{\mathcal{M}} d^{4} x \widetilde{\eta}^{a b c d} e_{a}^{I} e_{b}^{J} F_{c d I J}^{-}
$$

where now $e_{a}^{I}$ is a genuine tetrad field and $I=0, \ldots, 3$ are $\mathrm{SO}(4)$ indices, $F_{a b}^{I J-}$ is the curvature of an anti-self-dual connection $A_{a}^{-I J}$ defined by $F_{a b}^{I J-}=2 \partial_{[a} A_{b]}^{-I J}+2 A_{[a}^{-I K} A_{b] K}^{-}{ }^{J}$. Following Ref. [9] we write

$$
A_{a}^{-I J} \equiv\left[\begin{array}{cc}
0 & A_{a}^{j} \\
-A_{a}^{i} & -\epsilon^{i j k} A_{a k}
\end{array}\right], \quad e_{a}^{I} \equiv\left[\begin{array}{cc}
-\frac{1}{2} v_{a} & e_{a}^{i}
\end{array}\right],
$$

so that Eq. (4) becomes

$$
S=\frac{1}{2} \int_{\mathcal{M}} d^{4} x \widetilde{\eta}^{a b c d}\left[v_{a} e_{b}^{i} F_{c d i}+\epsilon^{i j k} e_{a i} e_{b j} F_{c d k}\right] .
$$

As we can see the (anti)-self-dual action can be obtained by adding a term involving a one-form field $v_{a}$ to the usual Husain-Kuchar action (1). A full discussion of Eq. (5) can be found in Ref. [9].

In the view of the previous formula it is natural to wonder what happens if instead of taking $v_{a}$ as a general one-form one considers it to be the gradient of a scalar $\nabla_{a} \phi$. Do we still have GR or something else? Let us consider then the following action:

$$
\hat{S}=\frac{1}{2} \int_{\mathcal{M}} d^{4} x \widetilde{\eta}^{a b c d}\left[-e_{a}^{i} F_{b c i} \nabla_{d} \phi+\epsilon^{i j k} e_{a i} e_{b j} F_{c d k}\right] .
$$

Before attempting to unravel its physical meaning, some preliminary remarks are in order. First of all the action is no

\footnotetext{
${ }^{3}$ Diffeomorphisms are actually generated by a linear combination of the Gauss law and the vector constraint.

${ }^{4} \mathrm{We}$ actually use anti-self-dual fields for calculational purposes.
}

longer $\mathrm{SO}(4)$ invariant ${ }^{5}$ although it is obviously $\mathrm{SO}(3)$ invariant. Second, we see now that $S$ is linear in the time derivatives of $\phi$ so we expect to have a scalar constraint linear in its canonically conjugate momentum (that after quantization will lead to a Schrödinger type of equation). It is natural to wonder if Eq. (6) could be an action for gravity (with an explicit time variable given by the scalar field $\phi$ ). The answer turns out to be in the negative though, at the end of the day, one discovers that Eq. (6) is still interesting in its own right. In order to check whether Eq. (6) describes GR or not we consider the field equations coming from Eq. (4) (remembering that we take now $e_{a}^{0}=\nabla_{a} \phi$ ). The field equation obtained by varying with respect to $A_{I J}^{-}$is

$$
\left[\nabla_{[a}\left(e_{b}^{I} e_{c]}^{J}\right)\right]^{-}=0
$$

From Eq. (7) we find out immediately that $A_{I J}^{-}$is equal to the anti-self-dual part of the $\mathrm{SO}(4)$ connection $\Gamma_{a}^{I J}$ compatible with $e_{a}^{I}$ defined by

$$
\mathcal{D}_{a} e_{b}^{I} \equiv \partial_{a} e_{b}^{I}-\Gamma_{a b}^{c} e_{c}^{I}+\Gamma_{a}^{I K} e_{b K}=0,
$$

where $\Gamma_{a b}^{c}$ is the Christoffel symbol of the four-metric $g_{a b} \equiv e_{a}^{I} e_{b I}$. Notice that, generically, the determinant of $e_{a}^{I}$

$$
\operatorname{det} e_{a}^{I}=\frac{1}{3 !} \widetilde{\eta}^{a b c d}\left(\nabla_{a} \phi\right) \epsilon^{i j k} e_{b i} e_{c j} e_{d k}
$$

is different from zero so that we can invert Eq. (8) to write $\Gamma_{a}^{I J}$ in terms of $e_{a}^{I}$ and its derivatives. By substituting $A_{a}^{-I J}=\Gamma_{a}^{-I J}[e, \phi]$ back into Eq. (4) we get

$$
S=\int_{\mathcal{M}} d^{4} y \sqrt{g[e, \phi]} R[e, \phi],
$$

where $R$ is the scalar curvature of $g_{a b} \equiv e_{a}^{I} e_{b I}=\nabla_{a} \phi \nabla_{b} \phi$ $+e_{a}^{i} e_{b i}$. If, by choosing $e_{a}^{i}$ and $\phi$ we can generate arbitrary and noncorrelated $g_{a b}[e, \phi](x)$ and

$\delta g_{a b}[e, \phi](x)=\int_{\mathcal{M}} d^{4} y\left[\frac{\delta g_{a b}(x)}{\delta e_{c}^{i}(y)} \delta e_{c}^{i}(y)+\frac{\delta g_{a b}(x)}{\delta \phi(y)} \delta \phi(y)\right]$

then $S$ must be an action for full GR, otherwise, it is something else. At a certain point with coordinates $x$ it is indeed true that both $g_{a b}$ and $\delta g_{a b}$ can be chosen to be anything we want. However, it is not clear that the same conclusion is true for all the points in a neighborhood of $x$ due to the restrictions that we have imposed to the form of some of the components of the tetrads (in fact the main result of the paper shows that $g_{a b}$ and $\delta g_{a b}$ are not completely arbitrary in all the points of $\Sigma$ ).

\footnotetext{
${ }^{5}$ Because the gradient of a scalar function does not transform as the zero component of a $\mathrm{SO}(4)$ vector [9].
} 


\section{HAMILTONIAN FORMULATION FOR THE NEW ACTION}

By introducing a foliation as in Sec. II we can write

$$
\begin{aligned}
\hat{S}= & \int d t \int_{\Sigma} d^{3} x\left\{\dot{A}_{a}^{i} \widetilde{\eta}^{a b c}\left[\epsilon_{i j k} e_{b}^{j} e_{c}^{k}-e_{b i} \nabla_{c} \phi\right]\right. \\
& +A_{0}^{i} \nabla_{a}\left[\widetilde{\eta}^{a b c}\left(\epsilon_{i j k} e_{b}^{j} e_{c}^{k}-e_{b i} \nabla_{c} \phi\right)\right]+\frac{1}{2} \dot{\phi} \widetilde{\eta}^{a b c} e_{a}^{i} F_{b c i} \\
& \left.+e_{0}^{i} \widetilde{\eta}^{a b c}\left[\epsilon_{i j k} e_{a}^{j} F_{b c}^{k}-\frac{1}{2} F_{a b i} \nabla_{c} \phi\right]\right\} \equiv \int d t L(t) .
\end{aligned}
$$

We denote $\widetilde{\pi}_{i}^{a}(x), \widetilde{\pi}_{i}(x), \widetilde{\sigma}_{i}^{a}(x), \widetilde{\sigma}_{i}(x)$, and $\widetilde{p}(x)$ the momenta canonically conjugate to $A_{a}^{i}(x), A_{0}^{i}(x), e_{a}^{i}(x), e_{0}^{i}(x)$, and $\phi(x)$ (with Poisson brackets given symbolically by $\{q, p\}=1)$. We find the following primary constraints:

$$
\begin{gathered}
\widetilde{\pi}_{i}^{a}+\widetilde{\eta}^{a b c}\left[e_{b i} \nabla_{c} \phi-\epsilon_{i j k} e_{b}^{j} e_{c}^{k}\right]=0, \\
\widetilde{\pi}_{i}=0, \\
\widetilde{\sigma}_{i}^{a}=0, \\
\widetilde{\sigma}_{i}=0, \\
2 \widetilde{p}-\widetilde{\eta}^{a b c} e_{a}^{i} F_{b c i}=0 .
\end{gathered}
$$

The Hamiltonian and the total Hamiltonian are

$$
\begin{aligned}
H= & \int_{\Sigma} d^{3} x\left\{e_{0}^{i} \widetilde{\eta}^{a b c}\left[\frac{1}{2} F_{a b i} \nabla_{c} \phi-\epsilon_{i j k} e_{a}^{j} F_{b c}^{k}\right]\right. \\
& \left.+A_{0}^{i} \nabla_{a}\left[\widetilde{\eta}^{a b c}\left(e_{b i} \nabla_{c} \phi-\epsilon_{i j k} e_{b}^{j} e_{c}^{k}\right)\right]\right\}, \\
H_{T}= & H+\int_{\Sigma} d^{3} x\left\{\lambda_{a}^{i}\left[\widetilde{\pi}_{i}^{a}+\widetilde{\eta}^{a b c}\left(e_{b i} \nabla_{c} \phi-\epsilon_{i j k} e_{b}^{j} e_{c}^{k}\right)\right]\right. \\
& \left.+\lambda^{i} \widetilde{\pi}_{i}+\mu_{a}^{i} \widetilde{\sigma}_{i}^{a}+\mu^{i} \widetilde{\sigma}_{i}+\zeta\left[2 \widetilde{p}-\widetilde{\eta}^{a b c} e_{a}^{i} F_{b c i}\right]\right\},
\end{aligned}
$$

where $\lambda_{a}^{i}(x), \lambda^{i}(x), \mu_{a}^{i}(x), \mu^{i}(x)$, and $\zeta(x)$ are arbitrary (at this stage) Lagrange multipliers. The conservation under the evolution defined by $H_{T}$ of the primary constraints (9)-(13) gives the secondary constraints

$$
\begin{aligned}
& \nabla_{a}\left[\widetilde{\eta}^{a b c}\left(e_{b}^{i} \nabla_{c} \phi-\epsilon^{i j k} e_{b j} e_{c k}\right)\right]=0, \\
& \widetilde{\eta}^{a b c}\left[\epsilon_{i j k} e_{a}^{j} F_{b c}^{k}-\frac{1}{2} F_{a b i} \nabla_{c} \phi\right]=0,
\end{aligned}
$$

and the following conditions on the Lagrange multipliers:

$$
\begin{gathered}
\tilde{\eta}^{a b c}\left[\left(\frac{1}{2} \delta_{i k} \nabla_{b} \phi+\epsilon_{i j k} e_{b}^{j}\right)\left(\mu_{c}^{k}-\nabla_{c} e_{0}^{k}-\epsilon^{k l m} e_{c l} A_{0 m}\right)\right. \\
\left.-\zeta \nabla_{b} e_{c i}-\epsilon_{i j k} e_{0}^{j} \nabla_{b} e_{c}^{k}\right]=0,
\end{gathered}
$$

$$
\begin{gathered}
\widetilde{\eta}^{a b c}\left[\left(\frac{1}{2} \delta_{i k} \nabla_{b} \phi-\epsilon_{i j k} e_{b}^{j}\right)\left(\lambda_{c}^{k}-\nabla_{c} A_{0}^{k}\right)\right. \\
\left.-\frac{1}{2} \zeta F_{b c i}+\frac{1}{2} \epsilon_{i j k} e_{0}^{j} F_{b c}^{k}\right]=0 \\
\widetilde{\eta}^{a b c}\left[\left(\mu_{a}^{i}-\nabla_{a} e_{0}^{i}-\epsilon^{i j k} e_{a j} A_{0 k}\right) F_{b c i}\right. \\
\left.+2\left(\lambda_{a}^{i}-\nabla_{a} A_{0}^{i}\right) \nabla_{b} e_{c i}\right]=0 .
\end{gathered}
$$

The conservation in time of Eqs. (16) and (17) does not generate new secondary constraints but only the following conditions on the Lagrange multipliers:

$$
\begin{gathered}
\widetilde{\eta}^{a b c}\left\{\nabla_{a}\left[\left(\frac{1}{2} \delta_{i k} \nabla_{b} \phi+\epsilon_{i j k} e_{b}^{j}\right) \mu_{c}^{k}\right]-\left(\nabla_{a} \zeta\right)\left(\nabla_{b} e_{c i}\right)\right. \\
\left.-\left(\frac{1}{2} \delta_{i k} \nabla_{a} \phi+\epsilon_{i j k} e_{a}^{j}\right) \epsilon^{k}{ }_{l m} \lambda_{b}^{l} e_{c}^{m}\right\}=0, \\
\widetilde{\eta}^{a b c}\left\{\left(\frac{1}{2} \delta_{i k} \nabla_{a} \phi-\epsilon_{i j k} e_{a}^{j}\right) \nabla_{b} \lambda_{c}^{k}-\frac{1}{2} \epsilon_{i j k} \mu_{a}^{j} F_{b c}^{k}\right. \\
\left.+\frac{1}{2} F_{a b}^{i} \nabla_{c} \zeta\right\}=0 .
\end{gathered}
$$

In principle, one expects that some combination of the second class constraints will be first class. The way to find out if this is the case is to solve the equations for the Lagrange multipliers. As we show in the Appendix it is possible to find $\mu_{a}^{i}$ from Eq. (18) and $\lambda_{a}^{i}$ from Eq. (19) and write them in terms of $\zeta, e_{a}^{i}, e_{0}^{i}, A_{a}^{i}$, and $A_{0}^{i}$ :

$$
\begin{aligned}
& \mu_{a}^{i}=\nabla_{a} e_{0}^{i}+\epsilon^{i j k} e_{a j} A_{0 k}+\underset{\sim a b}{\underset{{ }^{i}}{j}{ }^{j}} \widetilde{\eta}^{b c d}\left(\zeta \nabla_{c} e_{d}^{j}+\epsilon_{j k l} e_{0}^{k} \nabla_{c} e_{d}^{l}\right), \\
& \lambda_{a}^{i}=\nabla_{a} A_{0}^{i}-\frac{1}{2} \underset{\sim}{P_{b}{ }^{j}{ }^{i}} \tilde{\eta}^{b c d}\left(\zeta F_{c d j}-\epsilon_{j k l} e_{0}^{k} F_{c d}^{l}\right),
\end{aligned}
$$

where $\underset{\sim}{P_{a}{ }^{i}}{ }^{j}$ (which is calculated in the Appendix) satisfies

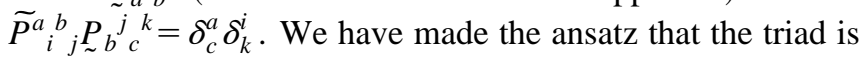
nondegenerate (and we will continue to do so throughout the paper). After some tedious algebra it is possible to verify that Eqs. (20)-(22) are identically satisfied by the previous $\mu_{a}^{i}$ and $\lambda_{a}^{i}$. We leave (some of) the details for the Appendix.

We want to stress here the importance of paying attention to the conditions on the Lagrange multipliers that appear in the Hamiltonian analysis. If one knows beforehand what a theory means, one can usually skip the arduous solution of the consistency equations as one does not need to know the explicit form of the Lagrange multipliers once all the first class constraints have been identified. However, it is true, in general, that the Lagrange multiplier equations themselves may imply additional constraints (they are nonhomogeneous linear equations) so, if one does not know the meaning of the theory one is dealing with, great attention must be paid to these equations in order to avoid missing some of the constraints and completely fail in the interpretation of the theory.

Substituting Eqs. (23), (24) in $H_{T}$ we get 


$$
\begin{aligned}
& H_{T}=\int_{M} d^{3} x\left\{e _ { 0 } ^ { i } \left[\widetilde{\eta}^{a b c}\left(\frac{1}{2} F_{a b i} \nabla_{c} \phi-\epsilon_{i j k} e_{a}^{j} F_{b c}^{k}\right)-\nabla_{a} \widetilde{\sigma}_{i}^{a}-\epsilon_{i j k} \widetilde{\sigma}_{l}^{a}{\underset{\sim}{a}{ }_{a}{ }^{j}{ }^{j}}_{\tilde{\eta}^{b c d}} \nabla_{c} e_{d}^{k}\right.\right. \\
& \left.-\frac{1}{2}\left[\widetilde{\pi}_{j}^{a}+\widetilde{\eta}^{a b c}\left(e_{b j} \nabla_{c} \phi-\epsilon_{j k l} e_{b}^{k} e_{c}^{l}\right)\right] \underset{\sim}{\underset{P}{{ }^{m}}{ }_{a}{ }^{j}} \widetilde{\eta}^{d e f} \boldsymbol{\epsilon}_{i m n} F_{e f}^{n}\right] \\
& -A_{0}^{i}\left(\nabla_{a} \tilde{\pi}_{i}^{a}+\epsilon_{i j k} e_{a}^{j} \widetilde{\sigma}^{a k}\right)+\lambda_{i} \tilde{\pi}^{i}+\mu_{i} \widetilde{\sigma}^{i} \\
& +\zeta\left[2 \widetilde{p}-\widetilde{\eta}^{a b c} e_{a}^{i} F_{b c i}+\widetilde{\sigma}_{i}^{a} \underset{\sim}{P}{ }_{a b}^{i}{ }^{j} \widetilde{\eta}^{b c d} \nabla_{c} e_{d j}\right.
\end{aligned}
$$

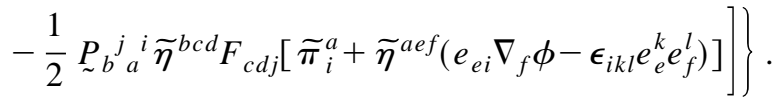

The terms proportional to $e_{0}^{i}$ and $A_{0}^{i}$ together give a first class Hamiltonian and the terms proportional to $\zeta, \lambda_{i}$, and $\mu_{i}$ are first class constraints (each of them). Of course, we have also all the remaining constraints provided by Eqs. (9), (10), (16), (17). The first class constraints $\widetilde{\pi}^{i}=0$ and $\widetilde{\sigma}^{i}$ $=0$ imply that $A_{0}^{i}$ and $e_{0}^{i}$ are arbitrary functions so we can just remove $\widetilde{\pi}^{i}=0$ and $\widetilde{\sigma}^{i}=0$ from Eq. (25). Furthermore, as now $A_{0}^{i}, e_{0}^{i}$, and $\zeta$ are arbitrary and $H_{T}$ is first class, the expressions that they multiply (linear combinations of first and second class constraints) must be first class constraints. In this way we get three sets of first class constraints plus the following independent second class constraints:

$$
\begin{gathered}
\widetilde{\pi}_{i}^{a}+\widetilde{\eta}^{a b c}\left[e_{b i} \nabla_{c} \phi-\epsilon_{i j k} e_{b}^{j} e_{c}^{k}\right]=0, \\
\widetilde{\sigma}_{i}^{a}=0 .
\end{gathered}
$$

These are very easy to deal with. In practice it is enough to remove $\widetilde{\sigma}_{i}^{a}$ from the first class constraints and write $e_{a}^{i}$ in terms of $\phi$ and $\tilde{\pi}_{i}^{a}$ by solving Eq. (26):

$$
\begin{aligned}
e_{a}^{i}= & \frac{1}{4 \tilde{\pi}} \eta_{a b c}\left\{ \pm\left[2 \tilde{\pi}-\left(\widetilde{\pi}_{l}^{d} \nabla_{d} \phi\right)^{2}\right]^{1 / 2} \epsilon^{i j k} \widetilde{\pi}_{j}^{b} \widetilde{\pi}_{k}^{c}\right. \\
& \left.-2\left(\tilde{\pi}_{k}^{d} \nabla_{d} \phi\right) \tilde{\pi}^{b k} \tilde{\pi}^{c i}\right\},
\end{aligned}
$$

where $\tilde{\tilde{\pi}} \equiv \operatorname{det} \tilde{\pi}_{i}^{a}$. The final Hamiltonian description is very simple. The phase space is coordinated by the canonically conjugate pairs ${ }^{6}\left(A_{a}^{i}, \widetilde{\pi}_{i}^{a}\right)$ and $(\phi, \widetilde{p})$ and the first class constraints are

$$
\begin{gathered}
\nabla_{a} \widetilde{\pi}_{i}^{a}=0, \\
\widetilde{\pi}_{i}^{b} F_{a b}^{i}+\widetilde{p} \nabla_{a} \phi=0, \\
\tilde{p}+\frac{1}{2}\left[2 \tilde{\tilde{\pi}}-\left(\widetilde{\pi}_{l}^{d} \nabla_{d} \phi\right)^{2}\right]^{-1 / 2} \epsilon^{i j k} \widetilde{\pi}_{i}^{a} \widetilde{\pi}_{j}^{b} F_{a b k}=0 .
\end{gathered}
$$

They are the Gauss law, which generates $\mathrm{SO}(3)$ gauge transformations, the vector constraint that (essentially) generates

${ }^{6}$ This is the symplectic structure given by the Dirac brackets. diffeomorphisms, and a scalar constraint linear in $\tilde{p}$. They are first class constraints. It is convenient to write them in "weighted" form

$$
\begin{gathered}
G\left(N^{i}\right)=\int_{\Sigma} d^{3} x N^{i} \nabla_{a} \widetilde{\pi}_{i}^{a}, \\
V\left(N^{a}\right)=\int_{\Sigma} d^{3} x N^{a}\left(\tilde{\pi}_{i}^{b} F_{a b}^{i}+\tilde{p} \nabla_{a} \phi\right), \\
S(N)=\int_{\Sigma} d^{3} x N\left\{\tilde{p} \overline{+} \frac{1}{2}\left[2 \tilde{\pi}-\left(\widetilde{\pi}_{l}^{d} \nabla_{d} \phi\right)^{2}\right]^{-1 / 2}\right. \\
\left.\times \epsilon^{i j k} \widetilde{\pi}_{i}^{a} \tilde{\pi}_{j}^{b} F_{a b k}\right\} .
\end{gathered}
$$

The three-dimensional diffeomorphisms are generated by the combination of the Gauss law and the vector constraint $D\left(N^{a}\right) \equiv G\left(N^{a} A_{a}^{i}\right)-V\left(N^{a}\right)$. We can write now the constraint algebra

$\left\{G\left(N^{i}\right), G\left(M^{i}\right)\right\}=G\left([N, M]^{i}\right)$, with $[N, M]^{i} \equiv \epsilon^{i j k} N_{j} M_{k}$,

$\left\{G\left(N^{i}\right), V\left(M^{a}\right)\right\}=0$,

$\left\{G\left(N^{i}\right), S(M)\right\}=0$,

$\left\{D\left(N^{a}\right), D\left(M^{b}\right)\right\}=D\left(-[N, M]^{a}\right)$, with $[N, M]^{a} \equiv N^{b} \partial_{b} M^{a}-M^{b} \partial_{b} N^{a}$,

$\left\{D\left(N^{a}\right), S(M)\right\}=S\left(-N^{a} \nabla_{a} M\right)$,

$\{S(N), S(M)\}=V\left[\left(N \partial_{a} M-M \partial_{a} N\right) \frac{4 \tilde{\pi}_{a}^{i} \widetilde{\pi}^{b i}}{2 \tilde{\pi}-\left(\tilde{\pi}_{l}^{d} \nabla_{d} \phi\right)^{2}}\right]$.

Several remarks are now in order. First, we see that the constraints are first class. As we have 20 canonical variables per space point in $\Sigma$ and seven first class constraints we have three degrees of freedom per space point-one more that in GR. Second, the Poisson bracket of the scalar constraint with itself closes and gives the vector constraint. This is in agreement with what one would expect from the arguments given by Hojman, Kuchar, and Teitelboim in Ref. [10] where they 
showed that the algebra of space-time deformations implied a constraint algebra of the type given by Eq. (30). Third, the structure of the scalar constraint is quite suggestive; it has two terms, one linear in $\widetilde{p}$ and another proportional to the scalar constraint in the Euclidean Ashtekar formulation for GR. This may signal a previously unnoticed relation between the Husain-Kuchař model and GR.

From Eqs. (28), (29) we can interpret the model very easily. It is enough to impose the gauge fixing condition $\phi=0$ [admissible because $\left.\{\phi(x), \widetilde{p}(y)\}=\delta^{3}(x, y)\right]$ to get rid of the scalar constraint and the $\phi$-dependent part of the vector constraint to recover the constraints of the usual HK model, namely,

$$
\begin{aligned}
& \nabla_{a} \tilde{\pi}_{i}^{a}=0, \\
& \widetilde{\pi}_{i}^{a} F_{a b}^{i}=0 .
\end{aligned}
$$

This means that in our formulation of the model the gauge orbits have one extra dimension so, in rigor, the models are equivalent only modulo gauge transformations. At this point the reader may have the temptation to think that, after all, it is trivial to add a scalar constraint to Eq. (31) in order to have a time variable (just take $\widetilde{p}=0$ and add the term necessary to generate diffeomorphisms on $\phi$ and $\tilde{p}$ to the vector constraint). The formulation thus obtained is, obviously, equivalent to ours [and can be derived from the action (6) by removing the derivatives of $\phi$ with an integration by parts]. However, it is much less obvious (and less trivial) the fact that with a suitable choice of a scalar constraint one gets, not only a time variable, but also a way to interpret the HK model as a theory for nondegenerate four-metrics at the Lagrangian level.

\section{THE FOUR-DIMENSIONAL PICTURE: NONDEGENERATE FOUR-METRICS}

The four-dimensional field equations coming from the action (6) are

$$
\begin{gathered}
\widetilde{\eta}^{a b c d}\left\{F_{a b i} \nabla_{c} \phi-2 \epsilon_{i j k} e_{a}^{j} F_{b c}^{k}\right\}=0, \\
\widetilde{\eta}^{a b c d}\left\{\nabla_{a} e_{b i} \nabla_{c} \phi+2 \epsilon_{i j k} e_{a}^{j} \nabla_{b} e_{c}^{k}\right\}=0, \\
\widetilde{\eta}^{a b c d}\left(\nabla_{a} e_{b}^{i}\right) F_{c d i}=0 .
\end{gathered}
$$

If we have a solution to these equations we can build a four-metric from the tetrad given by $\left(\nabla_{a} \phi, e_{a}^{i}\right)$ as $g_{a b}= \pm \nabla_{a} \phi \nabla_{b} \phi+e_{a}^{i} e_{b i}$. Notice that it is possible to write both Euclidean and Lorentzian four-metrics by choosing the sign in front of the $\nabla_{a} \phi \nabla_{b} \phi$ term. In general one expects that $g_{a b}$ is nondegenerate as can be checked by simply showing some solutions to Eqs. (32)-(34) such as

$$
A_{a}^{i}=0, \quad \phi=x^{0}, \quad e_{a}^{i}=\left[\begin{array}{lll}
0 & 0 & 0 \\
1 & 0 & 0 \\
0 & 1 & 0 \\
0 & 0 & 1
\end{array}\right] .
$$

As can be seen, Eq. (35) provides both the Euclidean and the Minkowski metric in $\mathbb{R}^{4}$. We see that we can solve the (ap- parent) paradox presented in the introduction by using the scalar field that is present now in the field equations to build nondegenerate four-metrics.

It is interesting at this point to compare the new equations (32) - (34) with the old ones (2). For starters we seem to have one more equation now than we had before; however, as we show below, this equation is not independent of the others and, also, any solution to Eq. (2) is a solution to it. In the following we use a procedure similar to the one that appears in Sec. III of Ref. [2]. Let us write

$$
\begin{gathered}
E_{a b}^{i} \equiv \nabla_{[a} e_{b]}^{i}, \\
\widetilde{n}^{a} \equiv \frac{1}{3 !} \widetilde{\eta}^{a b c d} \epsilon_{i j k} e_{b}^{i} e_{c}^{j} e_{d}^{k}, \\
\widetilde{\eta}_{i}^{a} \equiv-\frac{1}{2} \widetilde{\eta}^{a b c d} \epsilon_{i j k} e_{b}^{j} e_{c}^{k} \nabla_{d} \phi .
\end{gathered}
$$

Now $\widetilde{n}^{a} \widetilde{n}^{b} E_{a b}^{i}=0$ implies that there must exist $\widetilde{E}_{j}{ }^{i}$ such that

$$
\widetilde{n}^{a} E_{a b}^{i}=e_{b}^{j}{\widetilde{E_{j}}}^{i} .
$$

Notice that $e_{a}^{i}$ satisfy $\widetilde{n}^{a} \widetilde{E}_{a}^{i}=0$ so that any linear combination of the $e_{a}^{i}$ such as $e_{a}^{j} \widetilde{E}_{j}{ }^{i}$ will also satisfy $\widetilde{n}^{a} \widetilde{E}_{j}{ }^{i} e_{a}^{j}=0$. By the same reasoning there must exist $\widetilde{F}_{j}{ }^{i}$ such that

$$
\widetilde{n}^{a} F_{a b}^{i}=e_{b}^{j} \widetilde{F}_{j}{ }^{i} .
$$

We define also (we suppose $\widetilde{n}^{d} \nabla_{d} \phi \neq 0$ )

$$
\begin{aligned}
& e^{k l} \equiv \frac{1}{\left(\widetilde{n}^{d} \nabla_{d} \phi\right)^{2}} \epsilon^{i j k} \widetilde{\eta}_{i}^{a} \widetilde{\eta}_{j}^{b} E_{a b}^{l}, \\
& f^{k l} \equiv \frac{1}{\left(\widetilde{n}^{d} \nabla_{d} \phi\right)^{2}} \epsilon^{i j k} \widetilde{\eta}_{i}^{a} \widetilde{\eta}_{j}^{b} F_{a b}^{l} .
\end{aligned}
$$

We can extract all the content from Eqs. (32)-(34) by multiplying the first two by

$$
\epsilon^{i j k} \widetilde{\eta}_{i}^{a} \widetilde{\eta}_{j}^{b} \widetilde{\eta}_{k}^{c}, \quad \epsilon^{i j k} \widetilde{n}^{[a} \widetilde{\eta}_{j}^{b} \widetilde{\eta}_{k}^{c]},
$$

and the scalar equation (34) by

$$
\epsilon^{i j k} \widetilde{n}^{[a} \widetilde{\eta}_{i}^{b} \widetilde{\eta}_{j}^{c} \widetilde{\eta}_{k}^{d]}
$$

(which is proportional to $\widetilde{\eta}^{a b c d}$ ).

The result that we obtain from Eq. (32) is that $F^{i j}=-\frac{1}{2}\left(f^{i j}-\frac{1}{2} \delta^{i j} f\right)$ and $f^{i j}$ is symmetric and from Eq. (33) that $E^{i j}=\frac{1}{2}\left(e^{i j}-\frac{1}{2} \delta^{i j} e\right)$ and $e^{i j}$ is symmetric, where $e$ and $f$ are the traces of $e_{i j}$ and $f_{i j}$, respectively. In terms of $e^{i j}, f^{i j}, E^{i j}$, and $F^{i j}$ the scalar equation (34) gives $e^{i j} F_{i j}$ $+f^{i j} E_{i j}=0$; we see now that all the solutions to Eqs. (32) and (33) are solutions to the scalar equation and, hence, it is redundant.

If we consider now the standard HK equations we see that there is no scalar equation there. It is possible to extract the content of Eq. (2) by using the procedure introduced above. The only difference now is that we need an auxiliary scalar function (for example, the one that gives the foliation used in the passage to the Hamiltonian formulation) to define $\widetilde{\eta}_{i}^{a}$. 
We immediately find the result that appears in Ref. [2] $e^{[i j]}=0, f^{[i j]}=0, E^{i j}=0$, and $F^{i j}=0$, so that now it is also true that the scalar equation (34) is satisfied. In order to compare the solutions to Eqs. (2) and (32)-(34) one must take into account the new symmetry present in the model due to the introduction of $\phi$.

\section{FROM THE OLD TO THE NEW HUSAIN-KUCHAŘ MODEL: EQUIVALENCE AT THE LAGRANGIAN LEVEL}

Although we have seen from the Hamiltonian analysis that the new and all formulations of the HK model are strictly equivalent it is instructive to understand this from an independent point of view because the actions (1) and (6) look quite different [in fact one could claim that Eq. (6) is really "closer" to the self-dual action for GR than to the HK action].

The key idea to show this equivalence is the last result of the previous section, i.e., the fact that every solution to the ordinary HK equations (2) also satisfies Eq. (34). This means that nothing changes if we add this condition to the action (1) with a scalar Lagrange multiplier $\phi$. In this way we get

$$
\hat{S}_{1}=\int_{\mathcal{M}} d^{4} x \widetilde{\eta}^{a b c d}\left[-\phi F_{a b}^{i} \nabla_{c} e_{d i}+\epsilon_{i j k} e_{a}^{i} e_{b}^{j} F_{c d}^{k}\right],
$$

which is obviously equivalent to Eq. (6). Actually we can go even further. From the HK equations it is straightforward to show that Eq. (2) implies

$$
\widetilde{\eta}^{a b c d} \nabla_{a}\left(\epsilon_{i j k} e_{b}^{i} e_{c}^{j} e_{d}^{k}\right)=0
$$

so that even the action ${ }^{7}$

$$
\begin{aligned}
\hat{S}_{2}= & \int_{\mathcal{M}} d^{4} x \widetilde{\eta}^{a b c d}\left[\phi F_{a b}^{I} \nabla_{c} e_{d i}+\epsilon_{i j k} e_{a}^{i} e_{b}^{j} F_{c d}^{k}\right. \\
& \left.+\psi \epsilon_{i j k} \nabla_{a}\left(e_{b}^{i} e_{c}^{j} e_{d}^{k}\right)\right]
\end{aligned}
$$

describes the HK model. This last action admits an interesting interpretation. If we choose $\psi(x)=-(\Lambda / 3 !) \phi(x)$ with $\Lambda$ a real constant and consider the tetrad $e_{a}^{I} \equiv\left(\nabla_{a} \phi, e_{a}^{i}\right)$ whose inverse is given by

$$
e_{I}^{a} \equiv \frac{1}{\operatorname{det} e_{a}^{I}}\left[\begin{array}{c}
\tilde{n}^{a} \\
\widetilde{\eta}_{i}^{a}
\end{array}\right]
$$

with $\widetilde{n}^{a}$ and $\widetilde{\eta}^{a}$ as defined in the previous section we see that the added term is, in fact,

$$
\int_{\mathcal{M}} d^{4} x \Lambda\left(\operatorname{det} e_{a}^{I}\right),
$$

that is, a cosmological constant term. This is the simplest (and trivial) instance of a matter coupling to the HK model using the, now available, nondegenerate four-metric.

An additional curious fact is that the previous term is equivalent to

\footnotetext{
${ }^{7}$ There are even more possibilities which we do not discuss here.
}

$$
\int_{\mathcal{M}} d^{4} x \Lambda\left(\operatorname{det} e_{a}^{I}\right) e_{J}^{a} e^{a J} \nabla_{a} \phi \nabla_{b} \phi
$$

i.e., the coupling of the $\phi$ field to the "nondegenerate HK model" as a free scalar. A Hamiltonian analysis of these last actions with a "cosmological constant" following the lines of Sec. IV shows their equivalence with the usual HK model.

\section{CONCLUSIONS AND PERSPECTIVES}

As we have shown in the paper it is possible to describe the Husain-Kuchar model with an action principle for nondegenerate metrics. We have accomplished this by introducing a scalar field in such a way that adds no new degrees of freedom. This scalar plays, in a sense, the role of a time variable not only because we have now a Hamiltonian constraint that is linear in its canonical momentum but also because it allows dynamics to be referred to it. Our proposal should be compared to those of other authors (especially Refs. [6] and [7]). In these papers a scalar field is included as a means to define quantum gauge invariant observables, quoting Rovelli "matter observables which can be used to dynamically determine surfaces, the areas of which, we can measure.' Our contribution in this respect is that we have managed to achieve this goal without introducing new degrees of freedom in the model. We find it quite appealing that in this process we get a nice interpretation of the scalar $\phi$ as time. Not only can we do this but also, as a side result, we have now the possibility of coupling ordinary matter to the model. This provides a type of theories that lie in between those that have a matter evolving in a nondynamical background and full GR. We think that a lot can be learned from looking at these theories; we plan to study them in the future. Notice, by the way, that we have the choice of coupling the matter fields to Euclidean or Lorentzian metrics, depending on the choice of the sign in the first term of the four-metric $g_{a b}= \pm \nabla_{a} \phi \nabla_{b} \phi+e_{a}^{i} e_{b i}$.

We want to remark at this point that not knowing beforehand what the meaning of the action (6) is, one should be very careful in order to avoid missing constraints crucial for the interpretation of the theory. That is why we have paid so much attention to the solution of the equations for the Lagrange multipliers. Also, we emphasize again the contradiction in claiming that the Husain-Kuchar model only allows for the existence of degenerate four-metrics whereas it is obviously an extension of both Euclidean and Lorentzian GR. We believe that we have clearly solved this seemingly paradoxical fact in the paper.

\section{ACKNOWLEDGMENTS}

The authors want to thank our colleagues G. Immirzi, J. Julve, J. Leon, and G. Mena for their useful comments on this paper. J.F.B.G. also wants to thank J. M. Martín García for a very enlightening discussion. J.F.B.G. and R.T. were supported by CSIC contracts.

\section{APPENDIX}

As we have said in the main text of the paper we have paid special attention to the solution of the Lagrange multi- 
plier equations (18)-(22). The strategy that we have followed is simple. First solve Eq. (18) for $\mu_{a}^{i}$ and Eq. (19) for $\lambda_{a}^{i}$ and plug the result into the remaining ones. The result that we have obtained (for nondegenerate triads) shows that once we write these Lagrange multipliers in terms of $\zeta, A_{0 i}$, $e_{0 i}, A_{a i}, e_{a i}$, and $\phi$ the remaining equations are identically satisfied.

In order to solve Eqs. (18) and (19) we need to compute the inverses (that we denote $\underset{\sim}{\underset{P}{P}{ }^{i} b^{j}}$ ) of the $9 \times 9$ matrices

$$
\widetilde{P}_{i k}^{a c}(x) \equiv \widetilde{\eta}^{a b c}\left(\frac{1}{2} \delta_{i k} \nabla_{b} \phi+\epsilon_{i j k} e_{b}^{j}\right)
$$

and its transpose

$$
\widetilde{P}_{k i}^{c}{ }_{i}(x) \equiv \widetilde{\eta}^{a b c}\left(-\frac{1}{2} \delta_{i k} \nabla_{b} \phi+\epsilon_{i j k} e_{b}^{j}\right),
$$

where ${ }^{a}{ }_{i}$ are "double indices" that take the nine different values that make these matrices $9 \times 9$. The best way to build their inverses is to explicitly solve the equation

$$
\widetilde{M}_{i j}^{a b} X_{b}^{j} \equiv \widetilde{\eta}^{a b c}\left(\delta_{i j} v_{c}+\epsilon_{i j k} e_{c}^{k}\right) X_{b}^{j}=\widetilde{J}_{i}^{a}
$$

First we introduce the inverse triad $e_{i}^{a}$ such that $e_{i}^{a} e_{a}^{j}=\delta_{i}^{j}$ and write $\widetilde{\eta}^{a b c}=\widetilde{e} \epsilon^{i j k} e_{i}^{a} e_{j}^{b} e_{k}^{c}$ (here $\widetilde{e}$ is the nonzero determinant of the triad). Introducing this in Eq. (A1), expanding, and using the notation

$$
\begin{gathered}
X_{i j} \equiv e_{i}^{a} X_{a j}, \quad X \equiv e_{i}^{a} X_{a}^{i}, \quad J_{i}^{a} \equiv \widetilde{J_{i}^{a}} / \widetilde{e}, \quad J_{i j} \equiv e_{a i} J_{j}^{a}, \\
J \equiv e_{a i} J^{a i}
\end{gathered}
$$

we get

$$
\epsilon^{l m n} e_{l}^{a} X_{m i} v_{n}+\epsilon^{l m k} \epsilon_{i j k} e_{l}^{a} X_{m}^{j}=J_{i}^{a}
$$

which, after multiplying by $e_{a l}$ transforms into an equation that only involves objects with internal indices:

$$
\epsilon_{l}^{m n} X_{m i} v_{n}+X \delta_{i l}-X_{i l}=j_{l i} .
$$

Let us now take the trace of Eq. (A2) and multiply it by $\epsilon_{i l p} v^{p}$ and by $v_{i} v_{l}$. We find the following three equations:

$$
\begin{gathered}
-\epsilon^{i j k} X_{i j} v_{k}+2 X=J, \\
X_{i j} v^{i} v^{j}-v^{2} X-\epsilon^{i j k} X_{i j} v_{k}=-\epsilon^{i j k} J_{i j} v_{k}, \\
X v^{2}-X_{i j} v^{i} v^{j}=J_{i j} v^{i} v^{j},
\end{gathered}
$$

where $v^{2} \equiv v_{i} v^{i}$. Adding Eqs. (A4) and (A5) and using Eq. (A3) gives

$$
X=\frac{1}{2}\left[J+\epsilon^{i j k} J_{i j} v_{k}-v^{i} v^{j} J_{i j}\right]
$$

This means that we know how to express $X$ in Eq. (A2) in terms of $J_{i j}$ and $v_{i}$. If we look now at how the indices in the remaining $X_{i j}$ appear we see that the $i$ index is at both the second and the first place. If we could find the way to have both $i$ indices at the second place the remaining equation would be very easy to solve by inverting a simple $3 \times 3$ matrix. To this end we need to know the expression for $X_{[i j]}$ in terms of $J_{i j}$. This can be computed by multiplying Eq. (A1) both by $v_{a}$ and $\epsilon_{i l m} e_{a}^{m}$ and eliminating tangent space indices as before. One gets

$$
X_{[i j]}=J_{[i j]}-\frac{1}{2} \epsilon_{i j k} J^{l k} v_{l} .
$$

Using this result in Eq. (A2) we have

$$
\begin{aligned}
X_{i}^{k}\left(\delta_{j k}-\epsilon_{j k l} v^{l}\right)= & \frac{1}{2} \delta_{i j}\left(J+\epsilon^{p q r} J_{p q} v_{r}-v^{p} v^{q} J_{p q}\right) \\
& +\epsilon_{i j k} J^{l k} v_{l}-J_{i j} .
\end{aligned}
$$

Multiplying Eq. (A6) by

$$
\frac{1}{1+v^{2}}\left[\delta_{n j}+v_{n} v_{j}+\epsilon_{n j s} v^{s}\right]
$$

and reintroducing the triads we finally get

$$
\begin{aligned}
M_{\sim}{ }_{a b}^{i j}= & \frac{1}{2 \widetilde{e}\left(1+v^{2}\right)}\left[\delta_{n l}+v_{n} v_{l}+\epsilon_{n l r} v^{r}\right] \\
& \times\left[\delta^{i l} \delta_{k}^{j}-2 \delta_{k}^{i} \delta^{j l}+\delta^{i l} \epsilon_{m k}^{j} v^{m}\right. \\
& \left.+2 \epsilon^{i l j} v_{k}-v_{k} v^{j} \delta^{i l}\right] e_{a}^{n} e_{b}^{k} .
\end{aligned}
$$

The inverses of $\widetilde{P}_{i j}^{a b}$ and its transpose are immediately obtained from Eq. (A7). With them it is possible to check by direct substitution that the consistency equations (20)-(22) are identically satisfied. In practice the best strategy to do this is the following. First, eliminate the tangent space indices by multiplying by suitable combinations of inverse triads, then use the constraints (16) and (17) in the form

$$
\begin{gathered}
{\left[-\frac{1}{2} \delta_{i k} \nabla_{a} \phi+\epsilon_{i j k} e_{a}^{j}\right] \widetilde{\eta}^{a b c} \nabla_{b} e_{c i}=0,} \\
{\left[+\frac{1}{2} \delta_{i k} \nabla_{a} \phi+\epsilon_{i j k} e_{a}^{j}\right] \widetilde{\eta}^{a b c} F_{b c i}=0 .}
\end{gathered}
$$

In order to check Eq. (21) it is very useful to use the following identity:

$$
\widetilde{\eta}^{a b c}\left(P_{a i}^{k} \epsilon_{k l m}-\hat{P}_{a k l} \epsilon_{m i}^{k}\right) e_{c}^{m} \Lambda_{b}^{l}=0,
$$

where

$$
\begin{aligned}
& P_{a i k} \equiv v_{a} \delta_{i k}-\epsilon_{i j k} e_{a}^{j}, \\
& \hat{P}_{a i k} \equiv v_{a} \delta_{i k}+\epsilon_{i j k} e_{a}^{j},
\end{aligned}
$$

and $\Lambda_{b}^{l}$ is arbitrary. 
[1] M. P. Ryan Jr. and L. C. Shepley, Homogeneous Relativistic Cosmologies (Princeton University Press, Princeton, 1975).

[2] V. Husain and K. Kuchar, Phys. Rev. D 42, 4070 (1990).

[3] J. F. Barbero G., Int. J. Mod. Phys. D 3, 379 (1994).

[4] J. Samuel, Pramana, J. Phys. 28, L429 (1987); T. Jacobson and L. Smolin, Phys. Lett. B 196, 39 (1987).

[5] A. Ashtekar, Phys. Rev. Lett. 57, 2244 (1986); Phys. Rev. D 36, 1587 (1987); Non Perturbative Canonical Gravity (Notes prepared in collaboration with R. S. Tate) (World Scientific, Singapore, 1991).
[6] V. Husain, Phys. Rev. D 47, 5394 (1993).

[7] C. Rovelli, Nucl. Phys. B405, 797 (1993).

[8] P. A. M. Dirac, Lectures on Quantum Mechanics, Belfer Graduate School of Science Monograph Series No. 2 (Yeshiva University Press, New York, 1964).

[9] J. F. Barbero G., Phys. Rev. D 51, 5498 (1995).

[10] S. Hojman, K. Kuchar, and C. Teitelboim, Ann. Phys. (N.Y.) 96, 88 (1976). 